\title{
A comparison of gastroenteritis in a general practice-based study and a community-based study
}

\author{
M. A. S. DE WIT ${ }^{1 *}$, L. M. KORTBEEK ${ }^{2}$, M. P. G. KOOPMANS ${ }^{3}$, C. J. DE JAGER ${ }^{1}$, \\ W. J. B. WANNET ${ }^{2}$, A. I. M. BARTELDS ${ }^{4}$ AND Y. T. H. P. VAN DUYNHOVEN ${ }^{1}$ \\ ${ }^{1}$ Department of Infectious Diseases Epidemiology, National Institute of Public Health and the Environment, \\ PO Box 1, 37208A Bilthoven, The Netherlands \\ ${ }^{2}$ Diagnostic Laboratory for Infectious Diseases and Perinatal Screening, National Institute of Public Health \\ and the Environment, PO Box 1,37208A Bilthoven, The Netherlands \\ ${ }^{3}$ Research Laboratory for Infectious Diseases, National Institute of Public Health and the Environment, PO \\ Box 1, 37208A Bilthoven, The Netherlands \\ ${ }^{4}$ Netherlands Institute of Primary Health Care, Utrecht, The Netherlands
}

(Accepted 5 August 2001)

\section{SUMMARY}

We compared gastroenteritis cases that consulted a general practitioner (GP) with those who did not in a community-based study and also with those in a GP-based study. We aimed to identify factors associated with consultation, and with inclusion of cases by GPs, and secondly to study the effects on the frequency of detection of pathogens. Furthermore, we estimated the under-ascertainment by GPs. Both studies were performed in The Netherlands in the same population in an overlapping time-period. Overall, $5 \%$ of community cases consulted a GP. Cases who consulted suffered from more severe episodes than non-consulting cases. Inclusion of cases by GPs, instead of a study team, caused a selection of more severe cases with more chronic symptoms. When extrapolating data from GP-based studies, it should be taken into account that, in general practice, gastroenteritis due to bacteria and Giardia lamblia is a relatively large proportion of that in the community and gastroenteritis due to Norwalk-like viruses is a relatively small proportion. The incidence of gastroenteritis in general practices was estimated between 14 and 35 per 1000 person years.

\section{INTRODUCTION}

Gastroenteritis is one of the most common diseases worldwide [1, 2], and up-to-date information on the incidence, causative pathogens and transmission routes is necessary to control it. A study in the community is the best method to gain knowledge of gastroenteritis as a whole, but is very costly and timeconsuming. In general practices, gastroenteritis patients can be recruited efficiently, but cases seeking medical care are not representative of all cases, and little is known about factors influencing the consultation behaviour of cases [3, 4]. If the selection of patients that present to a GP is known, information

* Author for correspondence. obtained in a general practice-based study can be extrapolated to the community. To study this selection directly, we compared data from a community-based study (CB study, Sensor) and a general practice-based study (GP study), which had been conducted partly in parallel and based on the same population. We studied factors associated with consultation and factors associated with inclusion of cases by a GP, compared to inclusion by the study team.

\section{METHODS}

Both the GP study and the CB study were performed in cooperation with the general practice network of The Netherlands Institute of Primary Health Care (NIVEL). The population registered at these sentinel 
Table 1. Severity score of gastroenteritis

\begin{tabular}{|c|c|c|}
\hline Symptoms & Score* & $\begin{array}{l}\text { Modified } \\
\text { score } \dagger\end{array}$ \\
\hline Fever $>37.5^{\circ} \mathrm{C}$ & 2 & 2 \\
\hline \multicolumn{3}{|l|}{ Vomiting in $24 \mathrm{~h}$} \\
\hline Yes & & 1 \\
\hline Once & 1 & \\
\hline $2-4$ times & 2 & \\
\hline 5 times or more & 3 & \\
\hline \multicolumn{3}{|l|}{ Duration of vomiting } \\
\hline 1 day & 1 & \\
\hline 2 days & 2 & \\
\hline 3 days or more & 3 & \\
\hline Nausea & 1 & 1 \\
\hline Abdominal pain & 1 & 1 \\
\hline Abdominal cramps & 1 & 1 \\
\hline \multicolumn{3}{|c|}{ Loose of frequent stools in $24 \mathrm{~h}$} \\
\hline $1-3$ times & 1 & 1 \\
\hline 4-5 times & 2 & 2 \\
\hline 6 or more times & 3 & 3 \\
\hline \multicolumn{3}{|c|}{ Duration of loose or frequent stools } \\
\hline 1-4 days & 1 & \\
\hline 5 days & 2 & \\
\hline 6 days or more & 3 & \\
\hline Blood in stool & 2 & 2 \\
\hline Mucous in stool & 1 & 1 \\
\hline Head ache & 1 & 1 \\
\hline Maximal score & 21 & 13 \\
\hline
\end{tabular}

* For comparison consulting and non-consulting CB-study cases.

$\dagger$ For comparison consulting cases CB-study and GP-study cases.

practices was representative of the Dutch population for age, gender, geographical region and degree of urbanization. For the GP study, all GPs (from 42-44 practices) enumerated consultations for gastroenteritis on weekly enumeration forms from January 1996 until January 2000. Additionally, in 30-35 practices a case-control study was performed from May 1996 until May 1999 [5]. Questionnaires and stool samples from cases and controls were collected on the day of consultation. The CB study was a prospective cohort study with a nested case-control study [6]. An agestratified sample of persons registered in the 29-31 participating general practices was invited to participate. Follow-up was from 14 December 1998 until 14 December 1999, in two consecutive cohorts of 6 months. All participants completed a base-line questionnaire at the start of the study, and cases and controls completed a questionnaire concerning shortterm risk factors. Also, a medical diary was kept by cases during the 4 weeks after enrolment in the case- control study, addressing symptoms, use of medication, bed rest, and absence from work or school, and use of the health-care system. Finally, stool samples were submitted by cases (on days 1, 8, 15 and 22) and controls (days 1 and 8).

The case definition of gastroenteritis used in the GP study was: at least three loose stools in $24 \mathrm{~h}$ or loose stools with two additional symptoms or vomiting with two additional symptoms. The additional symptoms could be diarrhoea, vomiting, nausea, fever, abdominal pain, abdominal cramps, blood in the stool, or mucous in the stool. A new episode could only start after a symptom-free period of 2 weeks. In the CB study the same case-definition was used, with one additional option: vomiting at least three times in $24 \mathrm{~h}$. This option was included, because stool samples were also tested for bacterial toxins, which are often associated with vomiting only.

\section{Statistical analysis}

We composed a severity score for gastroenteritis, partly analogous to the severity score of Vesikari used for rotavirus gastroenteritis (Table 1) [7]. Degree of urbanization was classified as 'high' (>2500 addresses per $\mathrm{km}^{2}$ ), 'intermediate' (500-2500), and 'low' $(<500)$. The highest educational level achieved by those aged 18 years and older, and by one of the parents for those aged 0-17 years, was used for the educational level and classified as 'low' (primary school, lower vocational or lower general secondary education), 'intermediate' (intermediate vocational or intermediate general secondary and higher general secondary education), and 'high' (higher vocational secondary education and university education).

Consulting and non-consulting cases in the $\mathrm{CB}$ study were compared to identify person- and illness related factors associated with consultation, by a $\chi^{2}$ test for categorical variables, and by Wilcoxon signed rank test for continuous variables, and logistic regression analyses for the multivariate analyses. Selection of variables in the regression model was done retrospectively and manually, based on the likelihood ratio. Self-reported GP consultations in the CB study were compared with consultations reported on the enumeration forms, and checked retrospectively by the GP based on the patient-record. Only consultations that could be confirmed by one or both of the above methods were used in the analyses.

Consulting cases in the CB study were compared with cases from the GP study to identify factors associated with inclusion of cases by GPs compared to 
inclusion by the study team. In the CB study, the distribution of variables and pathogens in the first and second cohort were adjusted to the age distribution of the Dutch population. This standardization was necessary because the age distribution of the cohort was not representative for the Netherlands due to the age-stratified sampling frame [8]. The case groups were compared by logistic regression. For the regression analyses, study was included as the determinant; and age and cohort were included as confounders in the logistic model. In the CB study, consultations were reported in the medical diary, which covered a time-period of 4 weeks starting at the onset of symptoms. Therefore, cases in the GP study that consulted more than 4 weeks after onset of symptoms were excluded in this comparison (168 cases). Also cases in the CB study included for the additional criterion in the case-definition (vomiting three or more times in $24 \mathrm{~h}$ ) were excluded (this had no effect, since none of the consulting cases was in fact included as this criterion). A modified severity score was used, because not all variables in the original score were available for cases in the GP study (Table 1).

The pathogens detected were not included in these comparisons, because this information is not known to the case or the GP at the time of consultation and can therefore not influence their decision. Nevertheless, the presence of pathogens will differ between the case groups because of their relation to symptoms and socio-demographic variables. Therefore, we compared the percentages positive for the different pathogens detected in cases in the GP study with the standardized percentages in all cases in the CB study. For the CB study, results from first and second stool samples are presented (collected on day 0 and day 7 of the episode, respectively), because the median time of sampling in the GP study was 6 days after the onset of symptoms. Because of low numbers for several pathogens, and the need to standardize percentages, the pathogen-specific results from the CB study should be interpreted as merely indicative values. Therefore no exact ratios are presented for pathogens in the $\mathrm{CB}$ study and in the GP study.

Under-ascertainment of enumeration was measured as the percentage of confirmed consultations in the CB study that were not enumerated. An exact binomial $95 \%$ confidence interval was calculated. The incidence of gastroenteritis for which a GP was consulted was estimated based on the CB study and the GP study. For this comparison, the incidence in the CB study was standardized for age and cohort. The incidence from the GP study was corrected for under-ascertainment. In addition, cases that were and were not enumerated were compared, to study whether any systematic selection had occurred. Comparison of categorical variables was done by $\chi^{2}$ test and continuous variables by one Wilcoxon signed rank test.

\section{RESULTS}

\section{Consultations in the CB study}

In the CB study, 1052 case episodes were observed. Of these, 774 were included in the case-control study and for 646 a medical diary was completed. A GP consultation was reported by the patient for 94 gastroenteritis episodes: 82 practice visits and 12 house calls. Of these, 61 consultations (55 practice visits and 6 house calls) were confirmed by the GP. This corresponds with $9.4 \%$ of all episodes, and $5 \%$ when standardized for age and cohort. Of the 58 consultations that took place in a week for which an enumeration form was received, 13 were enumerated, yielding an under-ascertainment of $78 \%$ (standardized for age and cohort $83 \%, 95 \%$ confidence interval 56-100\%). Additional to the 61 episodes for which a practice visit or a house call was made, a telephone consultation was reported for 59 episodes, and consultation of another GP was reported for 15 episodes. Telephone consultations and consultations of another GP were not included as consultations in the analyses.

\section{Comparison of consulting cases and non-consulting cases in CB study}

In the univariate analyses, the age group under 1 year had the highest consultation rate (Table 2). In the older age groups, the consultation rate was similar. Cases with an intermediate level of education tended to consult more than cases with a high or low level of education. A severity score of seven or more (Table 1) was positively related to consultation. Individual symptoms significantly related to GP consultation were fever, a frequency of stools of six or more in $24 \mathrm{~h}$, and a frequency of vomiting of once in $24 \mathrm{~h}$. The same tendency, but not significant, was found for cases that suffered from blood in the stool, and abdominal cramps. The total duration of the episode was longer for consulting cases (median 12 days) than for nonconsulting cases $(9$ days $)(P=0 \cdot 02)$. The median 
Table 2. Percentage of cases with a confirmed consultation for gastroenteritis from all cases with a diary in the CB study, by patient- and illness-related variables in the entire episode

\begin{tabular}{|c|c|c|c|c|c|c|c|}
\hline & $\begin{array}{l}\text { Confirmed } \\
\text { consultation }\end{array}$ & $\begin{array}{l}\text { Cases } \\
\text { with diary }\end{array}$ & $\begin{array}{l}\% \text { of } \\
\text { cases } \\
\text { consulting }\end{array}$ & $\begin{array}{l}\text { Univariate } \\
\text { OR }\end{array}$ & $95 \% \mathrm{CI}$ & $\begin{array}{l}\text { Multivariate } \\
\text { OR }\end{array}$ & $95 \% \mathrm{CI}$ \\
\hline Total & 61 & 646 & $9 \cdot 4$ & & & & \\
\hline \multicolumn{8}{|l|}{ Age in years } \\
\hline 0 & 29 & 182 & $15 \cdot 9$ & $1 \cdot 0$ & - & $1 \cdot 0$ & - \\
\hline $1-4$ & 21 & 253 & $8 \cdot 3$ & 0.5 & $0 \cdot 3-0 \cdot 9$ & $0 \cdot 5$ & $0 \cdot 3-0 \cdot 9$ \\
\hline $5-11$ & 7 & 119 & 5.9 & $0 \cdot 3$ & $0 \cdot 1-0 \cdot 8$ & $0 \cdot 3$ & $0 \cdot 1-0 \cdot 8$ \\
\hline $12-17$ & 1 & 15 & 6.7 & $0 \cdot 4$ & $0 \cdot 0-3 \cdot 0$ & $0 \cdot 4$ & $0 \cdot 0-3 \cdot 1$ \\
\hline $18-64$ & 1 & 51 & $2 \cdot 0$ & $0 \cdot 1$ & $0 \cdot 0-0 \cdot 8$ & $0 \cdot 1$ & $0 \cdot 0-0 \cdot 8$ \\
\hline$\geqslant 65$ & 2 & 24 & $8 \cdot 3$ & 0.5 & $0 \cdot 1-2 \cdot 2$ & $0 \cdot 4$ & $0 \cdot 1-2 \cdot 1$ \\
\hline \multicolumn{8}{|l|}{ Education } \\
\hline $1-4$ & 6 & 105 & $5 \cdot 7$ & $0 \cdot 4$ & $0 \cdot 2-1 \cdot 1$ & & \\
\hline $5-6$ & 30 & 247 & $12 \cdot 2$ & $1 \cdot 0$ & - & & \\
\hline $7-8$ & 24 & 288 & $8 \cdot 3$ & $0 \cdot 6$ & $0 \cdot 4-1 \cdot 1$ & & \\
\hline \multicolumn{8}{|l|}{$\begin{array}{l}\text { Symptoms in entire } \\
\text { episode (yes } v s . \text { no) }\end{array}$} \\
\hline Fever & 40 & 275 & $14 \cdot 6$ & $2 \cdot 8$ & $1 \cdot 6-4 \cdot 9$ & $2 \cdot 5$ & $1 \cdot 4-4 \cdot 4$ \\
\hline Abdominal cramps & 33 & 281 & $11 \cdot 7$ & $1 \cdot 6$ & $0 \cdot 9-2 \cdot 7$ & 1.9 & $1 \cdot 1-3 \cdot 3$ \\
\hline Blood in stool & 2 & 9 & $22 \cdot 2$ & $2 \cdot 8$ & $1 \cdot 6-13 \cdot 7$ & & \\
\hline \multicolumn{8}{|c|}{ Frequency of stools in $24 \mathrm{~h}$} \\
\hline 0 & 11 & 126 & 8.7 & $1 \cdot 0$ & - & & \\
\hline $1-3$ & 27 & 333 & $8 \cdot 1$ & 0.9 & $0 \cdot 4-1 \cdot 9$ & & \\
\hline $4-5$ & 14 & 142 & $9 \cdot 9$ & $1 \cdot 1$ & $0 \cdot 5-2 \cdot 6$ & & \\
\hline$\geqslant 6$ & 9 & 43 & $20 \cdot 9$ & $2 \cdot 8$ & $1 \cdot 1-7 \cdot 2$ & & \\
\hline \multicolumn{8}{|c|}{ Frequency of vomiting in $24 \mathrm{~h}$} \\
\hline No vomiting & 22 & 294 & $7 \cdot 5$ & $1 \cdot 0$ & - & & \\
\hline once & 19 & 131 & $14 \cdot 5$ & $2 \cdot 1$ & $1 \cdot 1-4 \cdot 0$ & & \\
\hline $2-4$ & 14 & 151 & $9 \cdot 3$ & $1 \cdot 3$ & $0 \cdot 6-2 \cdot 5$ & & \\
\hline$\geqslant 4$ & 6 & 68 & $8 \cdot 8$ & $1 \cdot 2$ & $0 \cdot 5-3 \cdot 1$ & & \\
\hline \multicolumn{8}{|l|}{ Severity score } \\
\hline $0-3$ & 6 & 137 & $4 \cdot 4$ & $1 \cdot 0$ & - & & \\
\hline $4-6$ & 19 & 233 & $8 \cdot 2$ & $1 \cdot 9$ & $0 \cdot 8-5 \cdot 0$ & & \\
\hline$\geqslant 7$ & 36 & 274 & $13 \cdot 1$ & $3 \cdot 3$ & $1 \cdot 4-8 \cdot 0$ & & \\
\hline
\end{tabular}

Only variables presented for which inclusion in the logistic regression model decreased the log-likelihood ratio significantly $(P<0 \cdot 1)$.

duration of frequent stools was significantly longer in consulting cases (5.5 days) than in non-consulting cases (4 days) $(P=0.03)$, as was the median duration for loose stools ( $6 v s .4$ days, $P<0.01)$, for abdominal pain ( 5 vs. 2 days, $P<0.01)$ and for fever ( 2 vs. 2 days, $P=0.01)$. Gender, country of birth, degree of urbanization, region, income, chronic abdominal symptoms, abdominal pain, nausea, vomiting, loose stools, mucous in stool, head ache, bed rest and absence from work or school showed no relation to consultation. In the multivariate logistic regression model only age, fever and abdominal cramps were independently associated with consultation.

When considering only the symptoms that occurred before consultation, only fever, and a frequency of stools of six or more were significantly associated with GP-consultation (not in table). The duration of symptoms at the time of consultation (4 days) was shorter than the total duration of the episode for nonconsulting cases $(P<0 \cdot 01)$.

\section{Comparison of consulting cases in CB study and cases in GP study}

Compared to consulting cases from the CB study, cases from the areas with the highest degree of urbanization were overrepresented in the GP study, as were cases from the western region of the country (Table 3). Chronic intestinal disorders ( $>1$ month) were more frequent in the GP study. The median 
Table 3. Comparison of cases in GP study and consulting cases in CB study by patient-and illness-related variables in the episode until GP consultation

\begin{tabular}{|c|c|c|c|c|c|c|c|}
\hline & \multicolumn{2}{|c|}{$\begin{array}{l}\text { GP study } \\
(n=710) \\
\text { Crude }\end{array}$} & \multicolumn{3}{|c|}{$\begin{array}{l}\text { Consulting cases in CB } \\
\text { study }(n=61) \\
\text { Crude }\end{array}$} & \multicolumn{2}{|c|}{ Logistic regression } \\
\hline & No. & $\%$ & No. & $\%$ & Standardized* $(\%)$ & OR univ. & $95 \% \mathrm{CI}$ \\
\hline Age in years & & & & & & n.r. & \\
\hline 0 & 32 & $4 \cdot 5$ & 29 & $47 \cdot 5$ & $14 \cdot 1$ & & \\
\hline $1-4$ & 123 & $17 \cdot 4$ & 21 & $34 \cdot 4$ & $35 \cdot 1$ & & \\
\hline $5-11$ & 59 & $8 \cdot 4$ & 7 & $11 \cdot 5$ & $18 \cdot 6$ & & \\
\hline $12-17$ & 28 & $4 \cdot 0$ & 1 & $1 \cdot 6$ & $3 \cdot 1$ & & \\
\hline $18-64$ & 407 & $57 \cdot 6$ & 1 & $1 \cdot 6$ & $19 \cdot 3$ & & \\
\hline$\geqslant 65$ & 57 & $8 \cdot 1$ & 2 & $3 \cdot 3$ & $9 \cdot 8$ & & \\
\hline Urbanization & & & & & & $3.0 \dagger$ & $1 \cdot 6-5 \cdot 9$ \\
\hline Low & 771 & $10 \cdot 8$ & 13 & $21 \cdot 3$ & $20 \cdot 3$ & & \\
\hline Intermediate & 501 & $70 \cdot 6$ & 43 & $70 \cdot 5$ & $71 \cdot 1$ & & \\
\hline High & 132 & $18 \cdot 6$ & 5 & $8 \cdot 2$ & $8 \cdot 6$ & & \\
\hline Western region & 244 & $34 \cdot 4$ & 16 & $26 \cdot 2$ & $16 \cdot 4$ & $2 \cdot 6$ & $1 \cdot 1-6 \cdot 3$ \\
\hline Chronic g.i. disorder $\S$ & 147 & $20 \cdot 9$ & 2 & $3 \cdot 4$ & $9 \cdot 9$ & $4 \cdot 9$ & $1 \cdot 1-22 \cdot 0$ \\
\hline Fever & 283 & $39 \cdot 9$ & 37 & $60 \cdot 7$ & $73 \cdot 0$ & $0 \cdot 6$ & $0 \cdot 4-1 \cdot 2$ \\
\hline Vomiting & 300 & $42 \cdot 3$ & 36 & $59 \cdot 0$ & $52 \cdot 1$ & $0 \cdot 7$ & $0 \cdot 4-1 \cdot 4$ \\
\hline Nausea & 430 & $60 \cdot 6$ & 22 & $36 \cdot 1$ & $40 \cdot 4$ & $1 \cdot 0$ & $0 \cdot 5-1 \cdot 9$ \\
\hline Abdominal pain & 537 & $75 \cdot 6$ & 30 & $49 \cdot 2$ & $58 \cdot 3$ & $0 \cdot 7$ & $0 \cdot 3-1 \cdot 5$ \\
\hline Abdominal cramps & 544 & $76 \cdot 6$ & 32 & $52 \cdot 5$ & $68 \cdot 7$ & $0 \cdot 7$ & $0 \cdot 4-1 \cdot 4$ \\
\hline Blood in stool & 82 & $11 \cdot 5$ & 1 & $1 \cdot 6$ & $5 \cdot 7$ & $6 \cdot 2$ & $0 \cdot 8-48 \cdot 3$ \\
\hline Mucous in stool & 241 & $33 \cdot 9$ & 9 & $14 \cdot 8$ & $15 \cdot 9$ & $4 \cdot 3$ & $2 \cdot 0-9 \cdot 6$ \\
\hline Loose stools & 702 & $98 \cdot 9$ & 54 & $88 \cdot 5$ & $92 \cdot 8$ & $7 \cdot 6$ & $2 \cdot 1-26 \cdot 7$ \\
\hline Max freq stools p.d. & & & & & & $2 \cdot 5 \dagger$ & $1 \cdot 4-4 \cdot 3$ \\
\hline Normal & 121 & $17 \cdot 9$ & 14 & $23 \cdot 0$ & $10 \cdot 5$ & & \\
\hline $1-3$ stools & 77 & $11 \cdot 4$ & 21 & $34 \cdot 4$ & $58 \cdot 3$ & & \\
\hline $4-5$ stools & 162 & $24 \cdot 0$ & 15 & $24 \cdot 6$ & $20 \cdot 7$ & & \\
\hline$\geqslant 6$ stools & 316 & $46 \cdot 7$ & 11 & $18 \cdot 0$ & $10 \cdot 5$ & & \\
\hline
\end{tabular}

$\S \quad$ g.i., gastrointestinal (chronic: $>1$ month).

* Study population standardized according to the Dutch population.

$\dagger$ Proportional odds ratio presented.

$\$$ n.r., not relevant, because these were standardized to be equal.

duration of symptoms until consultation was significantly longer for cases in the GP study (6 days) than for consulting cases in the CB study (4 days) $(P<0 \cdot 01)$. The median value of the modified severity score (Table 1) was higher for cases in the GP study (6 vs. 4) $(P<0 \cdot 01)$. In general, all symptoms were more common in GP study cases than in consulting CBstudy cases, except fever and vomiting. Significantly more frequent in GP-study cases were mucous in the stool, loose stools and a higher frequency of stools.

\section{Pathogens in first and second stool samples in CB study and in the GP study}

Bacteria, predominantly Campylobacter sp. were overrepresented in the GP study compared to first and second samples in the CB study (Fig. 1). Rotavirus, astrovirus, and SLV were more frequent in GP-study cases than in second samples of CB-study cases, while the frequency in GP study cases was comparable to that in first samples of CB-study cases. NLV was less frequent in GP-study cases than in first and second samples of CB-study cases. Giardia was more frequent in GP-study cases than in first and second stool samples of CB-study cases. Cryptosporidium was equally frequent in both case groups. For most pathogens, the majority of positive second stool samples were from cases that also had a positive first stool sample, with the exception of NLV for which $42 \%$ of positive second samples were from cases that 


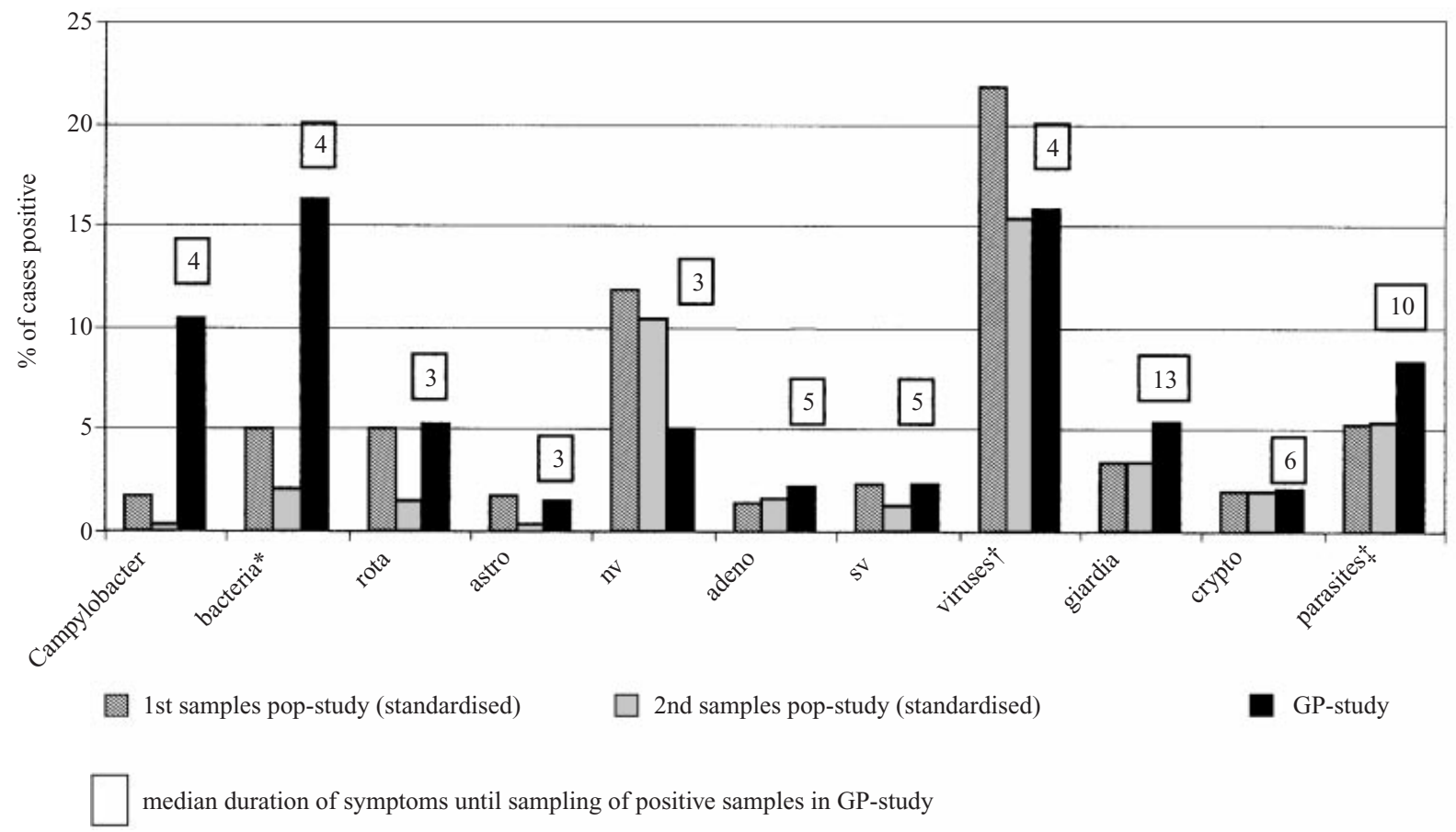

Fig. 1. Percentage of cases positive for the different (groups of) pathogens in first and second samples of the CB-study (standardized for age and cohort) and in the GP-study, and the median duration of symptoms until sampling of the samples positive for the different pathogens in the GP-study. *bacteria include Salmonella sp. Campylobacter sp. Yersinia sp. Shigella sp. and VTEC; †viruses include: rotavirus, adenovirus, astrovirus, SLV, NLV; łparasites include: Giardia lamblia, Cryptosporidium, Cyclospora, Entamoeba histolytica.

did not have a positive first sample. For Giardia lamblia this was $20 \%$.

\section{Comparison of enumerated cases and non-enumerated cases within CB study}

A comparison of the 13 consulting cases that were enumerated and the 45 cases that were not enumerated showed that cases with frequent stools were less often enumerated (14 vs. 55\%). Furthermore, cases that suffered from nausea were more often enumerated ( 35 vs. $13 \%$ ), and the percentage enumerated increased with decreasing degree of urbanisation (urban $0 \%$, urbanized $19 \%$, rural $39 \%$ ). No significant differences were found for other symptoms, age, gender, chronic intestinal symptoms, other chronic illnesses, presence of symptoms on the day of consultation, house calls versus practice visits, severity score, being under treatment of a specialist, number of GP visits in the past 3 months, and self-reported cause for symptoms.

\section{Incidence of gastroenteritis for which a GP was consulted}

The overall standardized incidence of gastroenteritis in the CB study was 283 per 1000 person years. In total, 30 case-episodes were included in the CB study based on the additional criteria of the case definition (vomiting at least three times in $24 \mathrm{~h}$ ); none of these had consulted a GP. After exclusion of these case episodes, the standardized incidence was 276 per 1000 person years, of which $5 \cdot 2 \%$ ( $9 \cdot 4 \%$ not standardized for age and cohort) reported a consultation that could be confirmed. This results in a standardized estimate of the incidence of gastroenteritis presented to a GP of 14 per 1000 person years. The incidence in the GP study, based on the enumeration forms was 5.9 per 1000 person years (corrected for list-inflation) [5]. However, adjusting this figure for the standardized estimate of under-ascertainment found in the $\mathrm{CB}$ study of $83 \%$ (not standardized for age and cohort: $78 \%$ ), gave an incidence of gastroenteritis for which a GP is consulted in the GP study of 35 per 1000 person years. The under ascertainment that could only be partially estimated in the GP study itself, gave a much lower estimate of $15 \%$, from which we arrived at the published incidence of 7.9 per 1000 person years [5].

\section{DISCUSSION}

\section{Consultation behaviour}

After standardization for age and cohort, for $5 \%$ of the gastroenteritis episodes a GP was consulted, of 
which $95 \%$ were practice visits and $5 \%$ house calls. This is lower than the estimate of $10 \%$ reported for the Netherlands in 1991, and the 17\% for England, but comparable to the $4 \%$ for Wales $[4,9,10]$. A significantly higher consultation rate was found in cases under 1 year of age, cases with fever, and cases with abdominal cramps. Also consultation was associated with high overall severity score and a longer duration of symptoms. This is consistent with other studies [11]. The fact that the under 1 year olds consulted more often than older persons might be explained by the fact that young children are subjectively and objectively more vulnerable than older cases. For instance, the risk of dehydration as a result of diarrhoea is higher in younger children. As expected, severity and duration of the episode were associated with consultation. However, the patients did not know the total duration of the episode at the time of consultation. Most likely, duration of symptoms is related to the severity, which is related to the decision of a patient to consult a GP. This is supported by the fact, that after correction for severity, the relation between duration of symptoms and consultation was no longer significant. The fact that the severity score was associated with the consultation rate shows that a rational measure of severity is indicative of the likelihood of consultation. Although studies on hospitalizations for gastroenteritis report an important role of social factors, studies on GP consultations mostly report the importance of severity and duration of symptoms [12-16]. Our results show that the assumption is to a large extent correct that studying cases in general practices means studying the more severe cases.

\section{Comparison of consulting cases in GP and CB study}

Cases in the GP study were more often from areas with a high degree of urbanization and from the western region of the country, than consulting cases from the CB study. This is caused by the fact that in the $\mathrm{CB}$ study, cases from these areas were already under-represented in the cohort [6].

In general, episodes of cases from the GP study were more severe and longer lasting than those of consulting cases in the $\mathrm{CB}$ study, indicating that inclusion by the GP leads to a more severe casepopulation than the overall consulting case-population. Furthermore, cases in the GP study more often suffered from chronic abdominal disorders than cases in the CB study. Because GPs know most of their patients, recruitment of cases for the study is influenced by their knowledge of the history of the patient, which might explain the high frequency of patients with chronic gastrointestinal symptoms. Additionally, inclusion of cases by the GP might have been more exclusive than by the study team. The selection that GPs make based on their professional view when including cases might exclude cases with gastrointestinal symptoms due to other illnesses and restrict the case group to pure gastroenteritis cases. In a study in the USA, approximately a quarter of the gastroenteritis episodes coincided with respiratory illness [12]. This might be one of the reasons that fever was more frequent in CB-study cases than in GPbased study cases. Although the selection that a GP makes when including cases might be a useful one, every GP will have a different view, and the selection criteria used by GPs are not clear. Therefore the use of a case definition for gastroenteritis in epidemiological studies remains the only method to ensure good knowledge of the population studied.

\section{Comparison of the proportion of cases attributable to different pathogens}

In the CB study, bacteria and viruses were detected less frequently in second stool samples than in first. This shows that by sampling after about 1 week, a proportion of these infections will be missed. Since sampling in a GP-based study does not take place until the patients consult a GP, which was after a median of 6 days of symptoms in our study, part of the diagnostic deficit can be attributed to the pathogens that were tested for. For parasites, the moment of sampling does not seem to influence the detection, which is consistent with the fact that parasites are excreted for a long time, but also indicates that the proportion of cases that does not start excreting until 1 week after the onset of symptoms is not substantial. However, in the GPstudy samples positive for Giardia lamblia were submitted after a median of 13 days and therefore, a valid comparison with the CB-study should be based on third stool sample (collected after 14 days). Surprisingly, for NLV, the detection decreased only slightly in second samples. The fact that a substantial amount of cases was not positive for NLV until the second sample indicates that excretion of NLV does not always start at the same time as the symptoms.

For Campylobacter sp., the proportion of GP-study cases was substantially higher than of CB-study cases, 
implying that a large percentage of cases associated with bacterial infections consulted a GP. This is consistent with the fact that Campylobacter sp. is associated with fever, blood in the stool, and a high frequency of stools [17], which are all symptoms that were found to be related to frequent consultation. NLV was more frequent in non-consulting cases than in consulting cases. This difference can not really be explained by the late moment of sampling in the GP study, but indicates that only a small percentage of NLV-related cases consulted a GP. This is consistent with a Canadian study in children that also reports NLV as the only viral pathogen detected more frequently in non-consulting children than in consulting children [18] The relatively short duration of episodes related to NLV, and the fact that it is not related to any of the symptoms for which consultations were more frequent, support this [17].

For rotavirus and to a lesser extent for astrovirus and SLV, the difference between first and second samples was substantial. Considering that the median time of sampling in the GP study for rotavirus, astrovirus, and SLV was 3-5 days, both the first and the second stool sample in the CB study should be used for a valid comparison of these pathogens in both studies. For astrovirus and SLV no information is available on whether this is a gradual decrease or a sudden drop somewhere in the first week. Therefore, for these viruses, a valid comparison with the detection in GP-study cases at 3-5 days after the onset of symptoms cannot be made. For rotavirus, a sharp decrease in the shedding of the virus was reported after 5 days [19]. Since rotavirus positive samples in the GP study were collected after a median of 3 days, a comparison with the first stool sample of the CB study seems more appropriate than with the second, resulting in a similar percentage attributable to rotavirus in both studies. This contradicts the results of Waters, who reported a higher percentage positive for rotavirus in consulting cases than in community cases [18]. For adenovirus and Cryptosporidium, no substantial differences can be seen between CB-study cases and GP-study cases.

In general, when studying cases in general practice, Campylobacter sp. and Giardia lamblia will be overrepresented, whereas NLV will be underrepresented. For the other pathogens, detection rates in general practice can be used as an estimate for those in the community. In a study in England, Salmonella sp. and Campylobacter sp. were also clearly more frequent in general practice-based cases than in community cases
[20]. The underrepresentation of NLV in general practices was not found in the English study. This difference might be a result of a lower threshold for GP consultation in England.

\section{Incidence of gastroenteritis for which a GP is consulted}

The incidence of gastroenteritis for which a GP was consulted in the CB study (14 per 1000 person years) was lower than that in the GP study adjusted for the under-ascertainment measured in the CB study (35 per 1000 person years). The estimate of underascertainment in the CB study might be an overestimate for the GP study, because GPs were more involved with the GP study and therefore probably more alert gastroenteritis. Also, normal practice in general practices might have been influenced by the fact that some consulting cases participated in the CB study, in spite of the fact that we focussed on not influencing the normal procedure in general practices by not giving any curative advice to cases. We should therefore conclude that the estimate of $83 \%$ underascertainment of the enumeration is not representative for the years before and after the CB study. Although, the $83 \%$ is most likely an over-estimate, underascertainment did occur. A comparison of the incidence of gastroenteritis for which a GP was consulted in the CB-study (14 per 1000 person years) with the crude incidence from the GP study (5.9 per 1000 person years), gives an estimate of underascertainment of $58 \%$. This might be due to the fact that the diagnosis of gastroenteritis by a GP is more exclusive than just the case definition, as was mentioned in the previous paragraph. In summary, the incidence of gastroenteritis for which a GP is consulted will be between 14 and 35 per 1000 person years, resulting in a total of 220000 to 560000 consultations for gastroenteritis per year in The Netherlands.

In The Netherlands, 1 in 20 cases with gastroenteritis consults a GP for this illness. As expected, consulting cases suffered from more severe gastroenteritis, especially with fever and abdominal cramps, and had longer episodes, than non-consulting cases. Besides, young children $(<1$ year of age) consulted more often. Inclusion of patients by the GP instead of the study team, leads to a selection of even more severe cases in the GP-based study and to the inclusion of more cases with chronic intestinal symptoms. In order to obtain comparable and complete data from 
different studies, general practitioners should use the case definition more strictly for enumeration and recruitment of cases for a study. When extrapolating data from GP-based studies, it would be taken into account that, in general practice, gastroenteritis due to bacteria and Giardia lamblia is a relatively large proportion of that in the community and gastroenteritis due to Norwalk-like viruses is a relatively small proportion.

\section{ACKNOWLEDGEMENTS}

We like to thank the participating general practitioners, Anita Suijkerbuijk, and Isabel Araya for their work in the data-collection, Denise Hoek, Jan Vinjé, Joke Admiraal, Hanneke Deijl, Miranda Asbroek, Jeroen Meijer, Nahid Nozari, Sandy Altena, and Petra de Bree for their assistance in the diagnostic testing, Nico Nagelkerke and Wilfrid van Pelt for their advice on the statistical analyses, and Frithjofna Abbink for her work on the design of the study.

\section{REFERENCES}

1. Guerrant RL, Hughes JM, Lima NL, Crane J. Diarrhoea in developed and developing countries: magnitude, special settings and aetiologies. Rev Infect Dis 1990; 12: S41-50.

2. Bern C, Martines J, Zoysa I de, Glass RI. The magnitude of the global problem of diarrhoeal disease: a ten-year update. Bull WHO 1992; 70: 705-14.

3. Borgdorff MW, Motarjemi Y. Surveillance of foodborne diseases: what are the options? WHO/FSF/ FOS/97.3. Food Safety Unit, World Health Organization, 1997.

4. Wheeler JG, Sethi D, Cowden JM, et al. Study of infectious intestinal disease in England: rates in the community, presenting to general practice, and reported to the national surveillance. BMJ 1999; 318: 1046-50.

5. Wit MAS de, Koopmans MPG, Kortbeek LM, Leeuwen WJ van, Bartelds AIM, Duynhoven YTHP van. Gastroenteritis in sentinel general practices in the Netherlands. Emerg Infect Dis. 2001; 7: 82-91.

6. Wit MAS de, Koopmans MPG, Kortbeek LM, et al. Sensor, a population-based cohort study on gastroenteritis in the Netherlands, incidence and aetiology. Am J Epidemiol. In press.

7. Ruuska T, Vesikari T. Rotavirus disease in Finnish children: use of numerical scores for clinical severity of diarrhoeal episodes. Scand J Infect Dis 1990; 22: 259-67.
8. Vademecum gezondheidsstatistiek Nederland 2000. Centraal bureau voor de Statistiek, Ministerie van Volksgezondheid, Welzijn en Sport. SDU's Gravenhage.

9. Wit MAS de, Hoogeboom-Verdegaal AMM, Goosen ESM, Sprenger MJW, Borgdorff MW. A populationbased longitudinal study on the incidence and disease burden of gastroenteritis and Campylobacter and Salmonella infections in four regions of the Netherlands. Eur J Epidemiol 2000; 16: 713-8.

10. Palmer S, Houston H, Lrevy B, Ribeiro D, Thomas P. Problems in the diagnosis of foodborne infections in general practice. Epidemiol Infect 1996; 117: 479-84.

11. Edwards A, Pill R. Pattern of help-seeking behavior for toddlers from two contrasting socio-economic groups: new evidence on a neglected topic. Fam Pract 1996; 13: 377-81.

12. Monto AS, Koopman JS. The Tecumseh study: occurrence of acute enteric illness in the community. Am J Epidemiol 1980; 112: 323-33.

13. McGee HM, Fitzgerald M. Childhood hospitalization for psychosocial reasons: the case of gastroenteritis. Int J Psychiatry Med 1999; 21: B55-68.

14. Thoren A, Lundberg O, Bergdahl U. Socio economic effects of acute diarrhea in adults. Scand J Infect Dis 1988; $20: 317-22$.

15. Nathwani D, Grimshaw J, Taylor RJ, Ritchie LD, Douglas JG, Smith CC. Factors influencing general practitioners' referral to hospital of adults with presumed infective diarrhoea. B J Gen Pract 1994; 44: $171-4$.

16. Conway SP, Newport MJ. Are all hospital admissions for acute gastroenteritis necessary? J Infect 1994; 29: 5-8

17. Wit MAS de, Koopmans MPG, Kortbeek LM, Leeuwen WJ van, Vinjé J, Duynhoven YTHP van. The etiology of gastroenteritis in sentinel general practices in the Netherlands. Clin Infect Dis $2001 ; 33$ : 280-8.

18. Waters V, Lee Ford Jones E, Petric M, Fearon M, Corey $\mathrm{P}$, Moineddein $\mathrm{R}$ and members of the pediactric rotavirus epidemiology study for immunization study group. Etiology of community-acquired pediatric viral diarrhea: a prospective longitudinal study in hospitals, emergency departments, pediatric practices and child care centers during the winter rotavirus outbreak, 1997 to 1998. Pediatr Infect Dis J 2000; 19: 843-8.

19. Haffejee IE. The pathophysiology, clinical features and management of rotavirus diarrhoea. Q J Med 1991; 79: 289-99.

20. Tompkins DS, Hudson MJ, Smith HR et al. A study of infectious intestinal disease in England: microbiological findings in cases and controls. Commun Dis Publ Hlth $1999 ; 2: 108-13$. 